\title{
Autophagy and signaling: their role in cell survival and cell death
}

\author{
P Codogno ${ }^{\star, 1}$ and AJ Meijer ${ }^{*, 2}$ \\ 1 INSERM U504, Glycobiologie et Signalisation cellulaire, Institut André Lwoff, \\ 16 avenue Paul-Vaillant-Couturier, 94807 Villejuif Cedex, France \\ 2 Department of Medical Biochemistry, Academic Medical Center, Meibergdreef \\ 15, 1105 AZ Amsterdam, The Netherlands \\ * Corresponding authors: P Codogno, INSERM U504, Glycobiologie et \\ Signalisation cellulaire, Institut André Lwoff, 16 avenue Paul-Vaillant- \\ Couturier, 94807 Villejuif Cedex, France. Tel: + 33 145595042; \\ Fax: + 33 146770233; E-mail: codogno@ vjf.inserm.fr and AJ Meijer, \\ Department of Medical Biochemistry, Academic Medical Center, Meibergdreef \\ 15, 1105 AZ Amsterdam, The Netherlands. Tel: + 31 205665154; \\ Fax: + 31 206915519; E-mail: a.j.meijer@amc.uva.nl
}

Received 04.5.05; revised 08.6.05; accepted 21.6.05

Edited by M Piacentini

\begin{abstract}
Macroautophagy is a vacuolar, self-digesting mechanism responsible for the removal of long-lived proteins and damaged organelles by the lysosome. The discovery of the ATG genes has provided key information about the formation of the autophagosome, and about the role of macroautophagy in allowing cells to survive during nutrient depletion and/or in the absence of growth factors. Two connected signaling pathways encompassing class-I phosphatidylinositol 3kinase and (mammalian) target of rapamycin play a central role in controlling macroautophagy in response to starvation. However, a considerable body of literature reports that macroautophagy is also a cell death mechanism that can occur either in the absence of detectable signs of apoptosis (via autophagic cell death) or concomitantly with apoptosis. Macroautophagy is activated by signaling pathways that also control apoptosis. The aim of this review is to discuss the signaling pathways that control macroautophagy during cell survival and cell death.

Cell Death and Differentiation (2005) 12, 1509-1518.

doi:10.1038/sj.cdd.4401751
\end{abstract}

Keywords: amino acids; macroautophagy; signal transduction; mTOR

\footnotetext{
Abbreviations: AICAR, 5-aminoimidazole-4-carboxamide riboside; AMPK, AMP-activated protein kinase; DAP kinase, deathassociated protein kinase; DAPK, death-associated protein kinase; DRP-1, death-associated related protein kinase-1; elF2 $\alpha$, eukaryotic initiation factor-2 alpha; 4E-BP1, eukaryotic translational initiation factor 4E-binding protein-1; ERK, extracellular signal-regulated protein kinase; FADD, Fas-associated death domain protein; GAIP, G alpha interacting protein; GFP, green fluorescent protein; LC3, light chain 3; 3-MA, 3-methyladenine;
}

MAPK, mitogen-activated protein kinase; mTOR, (mammalian) target of rapamycin; PDK1, phosphoinositide-dependent kinase-1; PI3K, phosphatidylinositol 3-kinase; PI(3)P, phosphatidylinositol 3-phosphate; $\mathrm{PI}(3,4) \mathrm{P}_{2}$, phosphatidylinositol 3,4 , bisphosphate; $\mathrm{PI}(3,4,5) \mathrm{P}_{3}$, phosphatidylinositol-3,4,5-trisphosphate; $\mathrm{PKB}$, protein kinase B; PKR, double-stranded RNA-activated protein kinase; PTEN, phosphatase and tensin homolog deleted from chromosome 10; Rheb, Ras homolog enriched in brain; RIP, receptor-interacting-protein; ROS, reactive oxygen species; S6, ribosomal protein S6; S6K or $\mathrm{p}^{\mathrm{S}} \mathrm{S}^{6 \mathrm{~K}}, 70 \mathrm{kDa}$ S6 kinase; TNF, tumor necrosis factor; TRAIL, TNF-related apoptosis-inducing factor; TSC, tuberous sclerosis complex

\section{Introduction}

Cells respond to changes in their environment and intracellular milieu by altering their anabolic and catabolic pathways. Two major pathways are involved in the catabolism of cellular material: the multi-enzyme proteasome system and autophagy, which culminates in lysosomal degradation. ${ }^{1,2}$ Both proteasome and autophagy are able to degrade proteins, but only autophagy can also degrade other macromolecules and even entire organelles. The term 'autophagy' includes several mechanisms, such as microautophagy, macroautophagy, and chaperone-mediated autophagy. This review is focused on macroautophagy (hereafter referred to as autophagy). Readers are referred to recent reviews of the other autophagic pathways. ${ }^{3,4}$ Autophagy is an evolutionary mechanism conserved in eukaryotic cells that starts with the formation of an autophagosome, enclosed within a double membrane that engulfs part of the cytoplasm. In mammalian cells, autophagosomes undergo a maturation process by fusing with endocytic compartments and lysosomes (for a review, see Eskelinen ${ }^{5}$ ).

The formation of the autophagosome depends upon Atg proteins, which were first discovered in yeast. ${ }^{6}$ Their role in autophagosome biogenesis has been recently reviewed. ${ }^{7}$

The molecular machinery is under the control of diverse signaling pathways, depending on the nature and the origin of the stimulus. ${ }^{8}$ In yeast, the kinase complex, Atg1, plays a central role in integrating signals downstream of the nutrient sensor TOR. ${ }^{9}$ Despite the fact that (mammalian) target of rapamycin (mTOR) is involved in the control of autophagy in mammalian cells, ${ }^{10}$ its connection with the molecular machinery remains to be clarified.

Autophagy is active at a basal level in most of the cells in the body, and this probably reflects its role in regulating the turnover of long-lived proteins, and getting rid of damaged structures. This activity is probably responsible for the antiaging role of autophagy. ${ }^{9,11}$ However, autophagy can be stimulated by various stress situations, including nutrient depletion. During periods of nutrient shortage, autophagy 
provides the constituents required to maintain the metabolism essential for survival. ${ }^{12}$

Autophagy is also implicated in various diseases, including cancer, neurodegenerative diseases, pathogen invasion, and muscle and liver disorders. ${ }^{13}$ In most of these situations, autophagy has both beneficial and harmful effects. One aspect of this complexity probably reflects the dual role of autophagy, which is both cell-protective and -destructive. The role of autophagy during cell death has been discussed in recent reviews. ${ }^{14,15}$ During apoptosis, the stimulation of autophagy can be either a protective mechanism or a process that contributes to cell death. In the absence of apoptosis, autophagy can trigger a form of cell death known as autophagic cell death or Type II programmed cell death, which is distinct from Type I programmed cell death (apoptosis). ${ }^{16}$

The aim of this review is to discuss the molecular control of autophagy, focusing particularly on signaling during protective autophagy, and during autophagy when the process is involved in the execution of a cell death program.

\section{Autophagy and Cell Survival}

As indicated in the previous section, in principle all eukaryotic cells are able to carry out autophagy; however, their autophagic capacity varies. It has long been assumed that autophagy is a nonspecific process, in which cytoplasmic structures and macromolecules are randomly sequestered in order to generate the oxidizable substrates and other molecules (e.g. amino acids) that are essential for cell survival when nutrients are scarce. However, the notion that autophagy was unspecific probably reflected the fact that substrate recognition had not been studied in great detail and we now know that, indeed, autophagy can also be very specific under certain conditions. Thus, the process can be involved in the elimination of damaged mitochondria ${ }^{17}$ or the selective removal of organelles that are functionally redundant (e.g. peroxisomes). ${ }^{13}$ As we will see, recent evidence suggests that the elimination of damaged mitochondria by autophagy may act as a rescue mechanism that the cell uses to escape from cell death, rather than as a mechanism producing cell death in its own right.

\section{Autophagy is activated when nutrients are deficient}

When cells lack essential nutrients, autophagy is activated to supply the missing components. A classical example is the mammalian liver, in which autophagy is switched on during starvation to produce amino acids which, after conversion into glucose, are used to meet the energy requirements of the brain and erythrocytes. Recent evidence indicates that autophagy in muscle tissue, although perhaps not as active as in hepatocytes, is quantitatively significant given the relatively large mass involved, also contributes to producing amino acids under these conditions. ${ }^{18,19}$ An interesting phenomenon, however, and one that has not been discussed in the literature so far, is that in mammals, after prolonged starvation (lasting for more than 3 days in man, for instance), autophagy slows down again because ketone bodies then begin to replace glucose at least to some extent, as fuel for the brain. Although it is obvious that this must occur to prevent excessive degradation of proteins that are essential for cell function, nothing is really known about the mechanism underlying the inhibition of autophagy under these extreme conditions (see also section 'What are the consequences for autophagy of downregulating amino-acid signaling?'). Of course, it is tempting to speculate that the ketone bodies themselves may be involved in regulating autophagy.

Autophagy is also extremely important as a source of oxidizable substrates in the neonate, which is suddenly faced by a sudden interruption of the supply of nutrients via the placenta, but which has not yet received sufficient nutrients via the milk. In an elegant study in mice expressing fluorescent green fluorescent protein (GFP)-tagged light chain 3 (LC3), an autophagosomal membrane marker, Kuma et al. ${ }^{18}$ recently showed that autophagy in the early neonatal period is transiently upregulated not only in the liver but also in many other tissues, including the heart, lung, diaphragm, pancreas, and muscle. Surprisingly, the increase in autophagy in the liver was relatively slight. However, it must be pointed out that, both in this study ${ }^{18}$ and in that carried out by Mizushima et al., ${ }^{19}$ in which LC3 was used to measure autophagy in various tissues in response to starvation, the level of autophagosomes, as measured using LC3-II, was not equivalent to flux through the autophagic system. This situation is entirely analogous to that of the metabolic pathways, in which changes in the steady-state concentrations of the pathway intermediates do not give any information about the flux through the pathway. It may very well be that the relatively low level of autophagosomes in the starved liver in vivo is attributable to a relatively high rate of elimination of autophagosomes rather than to a low rate of autophagosome formation.

Another situation in which autophagy is required to supply nutrients is that of cancer cells. Although suppression of autophagy may contribute to the initial rapid growth of tumors, in more advanced stages of cancer autophagy may be required to provide essential nutrients to the cells in the inner part of a solid tumor that do not have direct access to the circulation. ${ }^{20}$

Finally, autophagy can prevent cells from undergoing apoptosis by maintaining an adequate intracellular supply of substrates despite nutrient depletion ${ }^{21}$ or when the uptake of extracellular nutrients is inhibited by a lack of growth factor. ${ }^{22}$ For further reading on the role of autophagy in cell death, see the section 'Autophagy and cell death'.

\section{Inhibition of autophagy by amino acids}

Soon after the discovery of autophagy in the $1960 \mathrm{~s},{ }^{23}$ it became clear that amino acids, which are produced by autophagic protein breakdown, produce powerful feedback inhibition of autophagic sequestration, and that the process is also inhibited by insulin (in the liver) and activated by glucagon (for a review, see Mortimore et al. ${ }^{24}$ ). It is interesting to note that in the liver these hormones only affected autophagy at intermediate concentrations of amino acids, and not at either very low or very high concentrations of amino acids, when 
autophagic flux was maximal or minimal, respectively. ${ }^{24}$ As we will discuss later, this closely parallels the effect of insulin and glucagon and of amino acids on signal transduction.

Shortly after a report indicating that pharmacological inhibition of protein phosphatases inhibited autophagy in hepatocytes, ${ }^{25}$ an important step forward occurred in our understanding of the mechanism by which amino acids inhibit autophagy. This was the discovery that adding low concentrations of amino acids (leucine being particularly effective) to hepatocytes rapidly stimulated the phosphorylation of ribosomal protein S6 (S6), in synergy with either insulin or cell swelling induced by hypo-osmosis. ${ }^{10}$ Insulin alone did not stimulate S6 phosphorylation, and S6 phosphorylation induced by high concentrations of amino acids did not require the presence of insulin. Unlike insulin, glucagon inhibited S6 phosphorylation at intermediate concentrations of amino acids and stimulated proteolysis. Under various conditions, a linear relationship was found between the percentage inhibition of autophagic proteolysis (measured in the presence of cycloheximide to inhibit simultaneous protein synthesis) and the degree of phosphorylation of S6. ${ }^{10}$ Amino-acidinduced S6 phosphorylation was completely prevented by rapamycin, indicating that $\mathrm{mTOR}$ and p70S6 kinase were components of the signaling pathway. Rapamycin partly, but not completely, abolished the inhibition of autophagy by amino acids. Since rapamycin also produced a partial inhibition of protein synthesis in the absence of cycloheximide, it was proposed that the opposing processes of (global) protein synthesis and (autophagic) protein degradation may both be controlled by the same signaling pathway, which would be an efficient solution to metabolic regulation. ${ }^{10}$ The ability of rapamycin to stimulate autophagy has also been observed in other cell types, including yeast. ${ }^{13}$

Amino-acid stimulation of S6 phosphorylation was inhibited by the phosphatidylinositol 3-kinase (PI3K) inhibitors wortmannin or LY294002. ${ }^{26}$ Unexpectedly, and in contrast to the effect of rapamycin, these two compounds inhibited autophagy in the absence of amino acids. ${ }^{26}$ The explanation of this apparent anomaly was found by a study carried out with HT29 cells, a human colon cancer cell line. These experiments have shown that the product of class-III PI3K, phosphatidylinositol 3-phosphate $(\mathrm{PI}(3) \mathrm{P})$, is required for autophagy, whereas the products of class-I PI3K, phosphatidylinositol 3,4 , bisphosphate $\left(\mathrm{PI}(3,4) \mathrm{P}_{2}\right)$, and phosphatidylinositol-3,4,5trisphosphate $\left(\mathrm{PI}(3,4,5) \mathrm{P}_{3}\right)$, have inhibitory effects. ${ }^{27}$ Indeed, overexpression of phosphatase and tensin homolog deleted from chromosome 10 (PTEN), which removes the phosphate from the 3-position of $\mathrm{PI}(3,4) \mathrm{P}_{2}$ and $\mathrm{PI}(3,4,5) \mathrm{P}_{3}$, increases autophagy. ${ }^{28}$ The PI3K inhibitors do not distinguish between class-I and -III PI3K, and also inhibit the formation of $\mathrm{PI}(3) \mathrm{P}$, and thus inhibit autophagy. 3-Methyladenine (3-MA), the specific inhibitor of autophagy, turned out to be an inhibitor of PI3K, which explains why it has an inhibitory effect on both autophagy and S6 phosphorylation. ${ }^{26,27}$

Class-III PI3K (and its adaptor p150) was also found to be necessary for autophagy in yeast. This organism contains Vps34, a homolog of class-III PI3K, which is part of a complex that includes Vps15 (the homolog of p150 in mammalian cells), Vps30, and Atg14. ${ }^{29}$ This complex is required to recruit the Atg12-Atg5 conjugate to the preautophagosomal struc- ture. ${ }^{30}$ Interestingly, Beclin1, the mammalian homolog of Atg6, which is also required for autophagy, ${ }^{31}$ is found in a complex with class-III PI3K. ${ }^{32}$

The ability of amino acids to stimulate $\mathrm{S} 6$ phosphorylation (which reflects $70 \mathrm{kDa}$ S6 kinase (S6K) activity in situ) in hepatocytes in a rapamycin- and wortmannin-sensitive manner, and their synergy with insulin, not only provided a possible clue to a mechanism by which amino acids control autophagy but also was, in fact, the first demonstration that amino acids are able to stimulate insulin signaling. ${ }^{10} \mathrm{~A}$ few years later, this property of amino acids was confirmed for other insulin-sensitive cell types and, once again, leucine (but not the other branched-chain amino acids) was found to be the most effective amino acid. ${ }^{33}$ In addition to S6, downstream targets of mTOR, such as S6K, eukaryotic translational initiation factor 4E-binding protein-1 (4E-BP1), eukaryotic initiation factor-2 alpha (elF2 $\alpha$ ) kinase (the equivalent of general control nondepressible 2 (Gcn2) in yeast), and eEF2kinase, were found to be phosphorylated in response to amino-acid addition in a wortmannin-sensitive or LY294002sensitive manner. Direct evidence that amino acids can increase mTOR phosphorylation and activity was also obtained. ${ }^{34}$ At present, it is not clear whether the activation of mTOR by amino acids is direct, whether it involves the inhibition of tuberous sclerosis complex (TSC) $1 / 2$ (which inhibits mTOR), or whether the activation of mTOR occurs via decreased Ras homolog enriched in brain (Rheb) GTPase activity. ${ }^{35}$ Recent evidence indicates that it is unlikely that the inhibition of TSC2 by amino acids is the mechanism involved. $^{36}$

The idea that mTOR is involved in the negative control of autophagy is now generally accepted, ${ }^{13}$ but the mechanism by which this occurs is still largely unknown. In yeast, the inhibition of TOR may be required to stimulate ATG1 kinase activity, which is in turn required for the formation of the preautophagosomal membrane and also to increase the expression of ATG8, which is involved in the expansion step of the autophagosomal membrane. ${ }^{8}$ Further evidence in support of opposing regulatory effects on protein synthesis and autophagy by the amino-acid signaling pathway was obtained by demonstrating that two elF2 $\alpha$ kinases (GCN2 and PKR), TOR-downstream targets (see above; see also Figure 1), which regulate stress-induced translation arrest by phosphorylating the translation factor elF $2 \alpha$, positively control autophagic sequestration in yeast and mammalian cells in response to nutrient deprivation. ${ }^{37}$

There is a general consensus that amino acids do not directly affect the activity of protein kinase $B(P K B)$, and probably also do not affect class-I PI3K, two signaling proteins that are stimulated by insulin (for a review, see van Sluijters et $\left.a l^{33}\right)$. It is therefore likely that $\mathrm{mTOR}$ receives two parallel inputs, one via PI3K/phosphoinositide-dependent kinase-1 (PDK1)/PKB/TSC1,2/Rheb (stimulated by insulin and growth factors) and the other stimulated by amino acids. In addition, PDK1 can phosphorylate S6K directly (see Figure 1; for a detailed discussion of the various components of the pathway, see Jacinto and Hall ${ }^{38}$ ). Thus, both class-I PI3K (by insulin) and mTOR (by amino acids) must be activated to achieve full activation of mTOR downstream targets (for literature, see Meijer and Dubbelhuis ${ }^{39}$ ) and this would explain their 


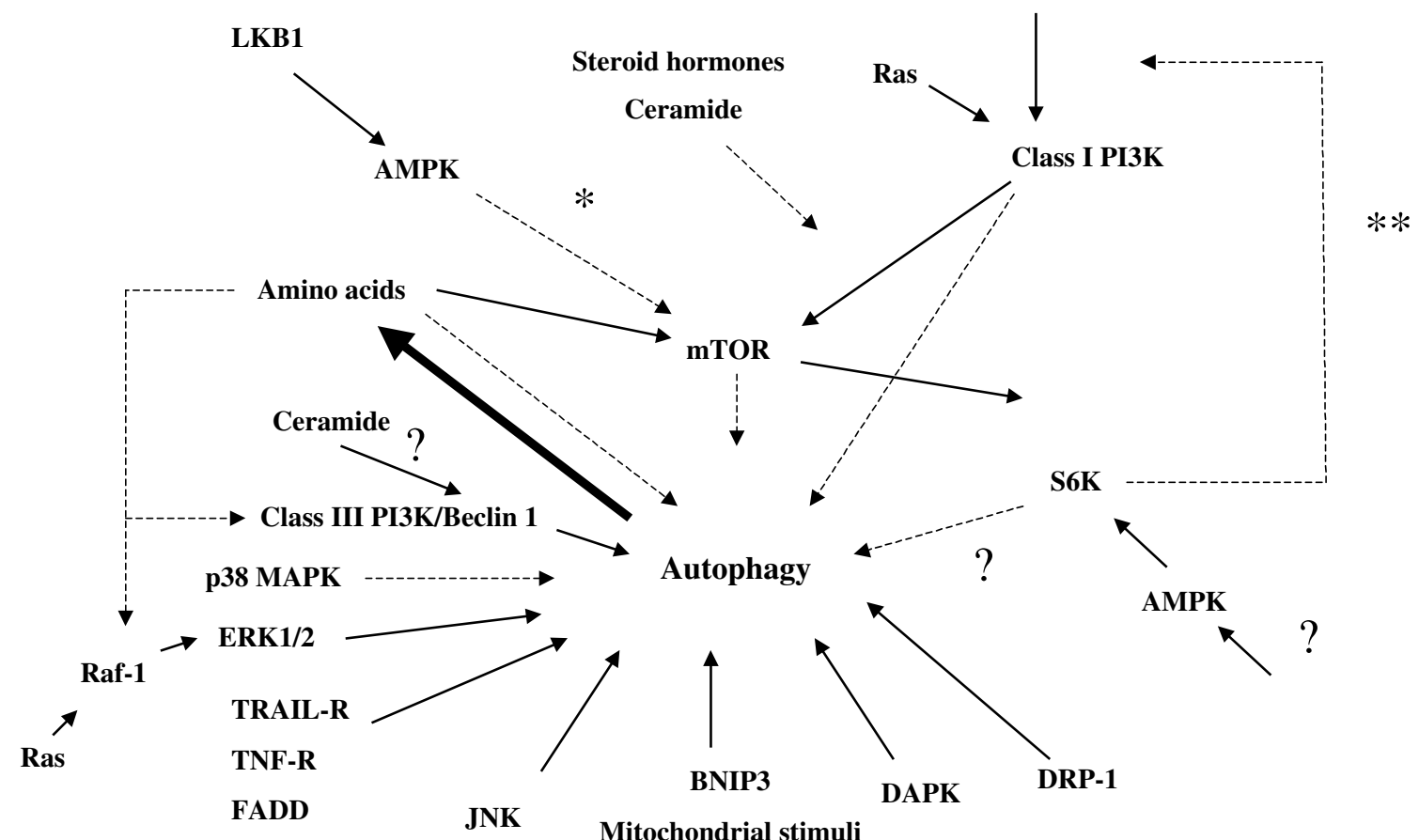

Mitochondrial stimuli

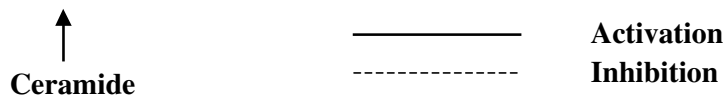

Figure 1 Diagram of autophagy signaling. Plain and dotted arrows represent activating and inhibiting signaling pathways, respectively. The outcomes of the various autophagy signaling pathways on cell survival and cell death are discussed in the main text. The thick arrow represents the recycling of amino acids produced by autophagic degradation. This recycling is highly active during starvation-induced autophagy. Amino-acid-dependent activation of mTOR plays a crucial role in repressing autophagy. However, mTOR-independent inhibition of autophagy by amino acids has also been reported. In addition, amino acids repress autophagy by interfering with Erk1/2 signaling, and with the activity of the class-III PI3K/beclin 1 complex involved in the formation of the autophagosome in intestinal and muscle cells, respectively. The inhibitory effect of the class-I PI3K signaling pathway on autophagy involves mTOR. However, it has been suggested in the literature that there is another signaling pathway that is independent of TOR. No role of the LKB1/AMPK signaling in controlling autophagy has yet been reported $\left(^{*}\right)$. Given the inhibitory effect of LKB1/AMPK signaling on mTOR signaling, we would have expected it to produce stimulation of autophagy. However, an inhibitory effect of AMPK, independent of LKB1, has been reported in rat hepatocytes. If a kinase is acting upstream of AMPK in this signaling process, its identity is not known. It has been suggested that AMPK-dependent S6K tail phosphorylation may have a structural role in inhibiting autophagy independently of its kinase activity (?). Inhibition of S6K activity is not involved in triggering autophagy, but this activity may repress autophagy by some unknown mechanism. In the fat body of Drosophila, S6K is required for autophagy to occur (not indicated in the figure). Inhibition of the insulin-signaling pathway by S6K as the result of a negative feedback loop has recently been demonstrated; however, the effect of this negative feedback on autophagy remains to be investigated $\left.{ }^{* *}\right)$. The ceramide-mediated expression of beclin 1 in human breast cancer cells in response to the antiestrogen tamoxifen remains to be elucidated

synergistic effects. In order to account for the ability of high concentrations of amino acids alone to activate p70S6 kinase or S6, in the absence of insulin, one has to assume that either the basal activity of class-I PI3K or a very slight stimulation of class-I PI3K by amino acids is sufficient to stimulate p70S6 kinase phosphorylation (for literature, see Meijer and Dubbelhuis ${ }^{39}$ ). In support of the scheme depicted in Figure 1, it has been found that overexpression of PKB inhibits autophagy, whereas expression of the dominant-negative PKB greatly stimulates the process. ${ }^{28}$

Although autophagy is negatively controlled by mTOR activity, the suggestion that the inhibition of autophagy by amino acids also proceeds via mTOR has been challenged.

In one study, ${ }^{40}$ carried out with hepatocytes isolated from rats fed a high-protein diet, rapamycin stimulated autophagy in the presence of insulin alone, but not in the presence of amino acids. As indicated above, in a variety of cell types, including hepatocytes, mTOR has an absolute requirement for amino acids, and is not activated by insulin alone. Sufficient amounts of amino acids from endogenous origin must apparently have been present in these studies (also see Beugnet et al. ${ }^{41}$ ). According to the authors, ${ }^{40}$ amino acids use an mTOR-independent pathway which is initiated by binding one or more amino acids, presumably leucine, to an aminoacid receptor protein in the plasma membrane, which then somehow leads to an inhibition of autophagy that is independent of mTOR. Although a separate signaling pathway starting at the plasma membrane cannot be excluded, the available evidence indicates that mTOR-dependent signaling is stimulated by intracellular amino acids, rather than extracellular amino acids (leucine in particular). ${ }^{41}$

In another study, carried out with $\mathrm{C} 2 \mathrm{C} 12$ myotubes, ${ }^{42}$ similar observations were made. Rapamycin was found to stimulate autophagic proteolysis, but could not abolish the 
stimulation of proteolysis by leucine starvation. The authors also concluded that amino acids (leucine in particular) do not inhibit autophagy via mTOR. It was suggested that amino acids could perhaps turn on different signaling pathways in different tissues, characterized by differences in their metabolism.

These studies clearly indicate that amino acids, as well as activating mTOR, can also inhibit autophagy independent of mTOR. What mechanisms could be responsible? One clue was provided by the finding that the induction of autophagy in C2C12 myotubes by amino-acid depletion was accompanied by an increase in Beclin1-associated class-III PI3K activity. ${ }^{43}$ Apparently, the production of $\mathrm{PI}(3) \mathrm{P}$ for autophagy is also controlled by amino acids, and is stimulated when amino-acid levels fall. Since changes in cell volume can affect molecular crowding, ${ }^{39}$ it may be speculated that amino-acid-induced cell swelling promotes the dissociation of the class-III Beclin1/ PI3K complex, and thus contributes to the inhibition of autophagy.

Another explanation for the inability of rapamycin to reverse amino-acid-induced inhibition of autophagy in some cell types is the recent demonstration that $\mathrm{mTOR}$ in mammalian cells occurs in two distinct complexes: one complex containing $\mathrm{mTOR}, \mathrm{G} \beta \mathrm{L}$, and raptor, which is rapamycin-sensitive, and another complex containing mTOR, $\mathrm{G} \beta \mathrm{L}$, and rictor, which is rapamycin-insensitive. ${ }^{44}$ Raptor mTOR phosphorylates S6K1 and is involved in controlling cell growth, whereas rictor mTOR is required for $\mathrm{Ser}^{473}$ phosphorylation of PKB and controls cell proliferation and survival. It is possible that the relative amount of mTOR sequestered in each of these complexes determines whether or not autophagy can be accelerated by rapamycin.

\section{Other signaling pathways stimulated by amino acids}

In human colon cancer HT-29 cells, another amino-aciddependent signaling pathway can control autophagy, in addition to the PI3K/mTOR pathway. Activation of Erk1/2 stimulates the GTPase-activating protein $\mathrm{G}$ alpha interacting protein (GAIP) and abolishes the inhibitory effect of trimeric Gi3 protein on autophagy (reviewed in Meijer and Codogno ${ }^{8}$ ). Amino acids, by stimulating the phosphorylation of $\mathrm{Ser}^{259}$, inactivate the Erk1/2 mitogen-activated protein kinase (MAPK) Raf-1 and downregulate autophagy. ${ }^{45}$ In contrast, in $\mathrm{C} 2 \mathrm{C} 12$ myotubes $^{43}$ the inhibition of autophagy by amino acids is not accompanied by any changes in Erk1/2 phosphorylation. Differences in amino-acid signaling mechanisms and in the control of autophagy may exist, apparently depending on the cell type, and perhaps also on the degree of differentiation. ${ }^{43}$

In the isolated, perfused rat liver and in cultured hepatocytes, amino-acid-induced cell swelling, due to the $\mathrm{Na}^{+}$. dependent concentrating influx of amino acids, inhibits autophagy because it activates $\mathrm{p} 38^{\mathrm{MAPK}}$; inhibition of this stress kinase prevents the inhibition of autophagy. Integrins appear to be involved in the osmosensing mechanism. ${ }^{46}$ In contrast, amino-acid-induced inhibition of autophagy in $\mathrm{C} 2 \mathrm{C} 12$ myotubes is independent of $\mathrm{p} 38^{\mathrm{MAPK}} .43$

\section{mTOR, energy, and autophagy}

Although it is now generally accepted that mTOR functions as an amino-acid sensor, ${ }^{38}$ recent evidence suggests that it also functions as a sensor of the cellular energy status. It was originally proposed that mTOR, because of its relative high $K_{m}$ for ATP, may respond to changes in the intracellular ATP concentration. ${ }^{47}$ However, it now seems more likely that changes in ATP are transmitted to mTOR by AMP-activated protein kinase (AMPK), because a small decline in cytosolic ATP results in a relatively large increase in the concentration of $\mathrm{AMP}$ via the adenylate kinase equilibrium. ${ }^{39}$ Activating AMP kinase inhibits mTOR-dependent signaling, and inhibits protein synthesis. ${ }^{39}$ This is in agreement with the function of AMPK to turn off ATP-dependent metabolic pathways. ${ }^{48}$ In this context, the association of mTOR with the mitochondrial outer membrane is noteworthy, because adenylate kinase is located in the mitochondrial intermembrane space, and mTOR is therefore ideally located to sense changes in the ATP/AMP ratio. ${ }^{39}$

In murine myotubes, $\mathrm{Ser}^{2448}$ of mTOR is phosphorylated in response to amino acids and growth factors; $\mathrm{Thr}^{2446}$ was identified as a novel phosphorylation site in mTOR. However, $\mathrm{Thr}^{2446}$ phosphorylation is activated by amino-acid deprivation or by AMPK. These two phosphorylation sites are mutually exclusive in that phosphorylation of one site inhibits phosphorylation of the other and vice versa. They may be viewed as switches, which integrate the counteracting signals of growth factors and nutrient deprivation. ${ }^{34}$

Since AMPK activation inhibits mTOR signaling, one would expect that, by analogy with the effect of rapamycin, AMP kinase activation would stimulate autophagy. Yeast cells contain the AMP kinase homolog, snf1p, which is, indeed, required for autophagy. ${ }^{49}$ In contrast, the activation of AMP kinase by 5-aminoimidazole-4-carboxamide riboside (AICAR) or by other pharmacological interventions in hepatocytes inhibits autophagy. ${ }^{50}$ In addition to its effects on mTOR phosphorylation (see previous paragraph), AMPK activation also results in the phosphorylation of $\mathrm{Thr}^{421}$ and $\mathrm{Ser}^{424}$ in the tail region of S6K1 in a rapamycin-insensitive manner; in contrast, amino acids stimulate the phosphorylation of $\mathrm{Thr}^{421}$ and $\mathrm{Ser}^{424}$ and $\mathrm{Thr}^{389}$ (required for S6K1 activity) in a manner sensitive to rapamycin. ${ }^{51}$ The physiological significance of the AMPK-induced phosphorylation of the tail region of S6K1 is not clear. It has been suggested, however, that S6K1 tail phosphorylation may convey a toxin-induced signal independently of S6K1 activity and this could perhaps lead to the suppression of autophagy. ${ }^{51}$

It must be stressed that so far the evidence that AMP kinase inhibits autophagy in hepatocytes rests entirely on data obtained using toxins or AICAR. The latter, after intracellular phosphorylation, is a nucleotide analog, and so we cannot rule out the possibility that AICAR has other effects: it may inhibit class-III PI3K, for example. In experiments carried out recently in our own laboratory with hepatocytes (unpublished observations), we observed that the antidiabetic agent metformin markedly activated AMPK, whereas autophagy remained largely unaffected. This would indicate that the activation of AMPK alone is not sufficient to inhibit autophagy. 
As indicated above, autophagy increases when cells have insufficient oxidizable substrate at their disposal, for example, inhibiting autophagy in apoptotic cells accelerates cell death, ${ }^{21}$ whereas glucose deprivation in a cardiomyocytederived cell line accelerates autophagy. ${ }^{52}$ In necrotic cells autophagy is increased. ${ }^{53}$ What could trigger autophagy under these conditions? It is questionable that in apoptotic cells, necrotic cells, or in cells without sufficient glucose, sufficient substrate will be available to maintain cellular ATP levels and to keep AMPK activity low. In addition, autophagy could specifically eliminate mitochondria with a low membrane potential. ${ }^{17}$ It is highly unlikely that AMP concentrations can be kept low in the vicinity of such mitochondria, especially in view of the fact that adenylate kinase is located in the mitochondrial intermembrane space, close to mitochondrial F1-ATPase. The inhibition of autophagy by AMPK, as proposed by Seglen et al. ${ }^{51}$ would in fact be counterproductive. The lifespan of Caenorhabditis elegans is extended by activating AMPK. ${ }^{54}$ As we will see later, it is the activation of autophagy and not its inhibition that increases the lifespan. In our opinion, we must seriously consider the possibility that AMPK, as in yeast, is required for autophagy to occur in mammalian cells, rather than inhibiting it. In this context, it is perhaps of significance that patients with Wolff/Parkinson/White syndrome, who have mutations in the $\gamma 2$ regulatory subunit of AMPK that result in overactivation of this enzyme, display a pronounced formation of vacuoles filled with glycogen within the myocyte, and suffer from cardiac hypertrophy and electrophysiological abnormalities. ${ }^{55}$ Of course, at present it is impossible to decide whether flux through the autophagic pathway actually increases or whether the processing of newly formed autophagosomes is blocked. Interestingly, inducing mutations similar to those found in these patients in the yeast homolog Snf4 protein stimulates AMPK activity in yeast cells. ${ }^{55}$

\section{What are the consequences for the autophagy of downregulating amino-acid signaling?}

Although amino acids and insulin act synergistically to activate mTOR-downstream targets, evidence is now accumulating that the overactivation of mTOR leads to the inhibition of the proximal part of the insulin-signaling pathway. ${ }^{56,57}$ This is because of phosphorylation of IRS1 by S6K, which results in decreased binding of the p85 regulatory subunit of PI3K to IRS1. As we have seen, class-I PI3K is required for mTORdownstream signaling, and this feedback system may be part of a homeostatic mechanism that is required to prevent the overactivation of mTOR by amino acids. It has even been proposed that the overactivation of mTOR could contribute to insulin resistance in obesity-linked diabetics. ${ }^{57}$ Downregulation of proximal insulin signaling can be expected to have consequences for autophagy, because a decline in inhibitory $\mathrm{PI}(3,4,5) \mathrm{P}_{3}$ will accelerate autophagy. Surprisingly, not much information is available about protein turnover in type II diabetes. Whether or not autophagy plays a role here is entirely unknown.

The possible consequences of feedback interaction of S6K on mTOR upstream signaling for autophagy was also suggested by $u^{58}$ in connection with a recent paper by
Scott et al. ${ }^{59}$ concerning the regulation of autophagy in the fat body of Drosophila melanogaster. In this study, the authors convincingly show that S6K is not inhibitory, as previously suggested, ${ }^{10}$ but, is in fact essential for autophagy. Scott et al. ${ }^{59}$ conclude that the inhibition of autophagy by Tor and the activation of S6K must be effected through different branches of the Tor pathway. Since the activation of S6K is Tor-dependent, it is assumed that in starvation, when Tor is switched off, S6K still needs to be activated for some time and that S6K downregulation may limit autophagy during prolonged starvation. ${ }^{59}$

Apparently, the same protein kinase (S6K) that is required for protein synthesis, an anabolic process, is also essential for autophagy, a catabolic process. ${ }^{59}$ This does not seem to be logical from the point of view of metabolic regulation. How can we solve this intellectual dilemma? Of course, we cannot exclude the possibility that Tor controls the synthesis of proteins required for expansion and maturation of the autophagosome, ${ }^{59}$ but this remains to be confirmed. An alternative explanation is the possibility of a negative feedback effect of S6K on signaling upstream of Tor, which would then increase autophagy as a result of the fall in class-I PI3K activity (see the previous section). This may be important, because even under nutrient-rich conditions cells must be able to carry out some autophagic activity, not in order to produce nutrients, which are plentiful, but in order to eliminate damaged cell structures or structures that are no longer needed by the cell. Conversely, when nutrients become scarce, for example, during starvation, the inactivation of Tor by a fall in amino-acid concentration accelerates autophagy, provided sufficient active S6K is still present (see above). During long-term starvation, S6K activity may fall so low that class -1 PI3K is activated again and this would then restrain excessive autophagy in order to prevent cell death, as suggested by Scott et al. ${ }^{59}$

Another possibility that has not been considered so far, is that tail-phosphorylated S6K, ${ }^{51}$ which is catalytically inactive, could have a structural role in autophagy, whereas S6K phosphorylated at $\mathrm{Thr}^{389}$ inhibits autophagy.

\section{Defective insulin signaling, autophagy, and lifespan}

In recent years, more and more evidence has been accumulating that defective insulin signaling extends the lifespan in various organisms, ranging from worms and flies to mammals. ${ }^{60}$ Presumably, this can be ascribed to accelerated autophagy. ${ }^{11,61}$ Calorie restriction also prolongs life and this, too, has been ascribed to increased autophagy. ${ }^{11}$ For decades defective mitochondria and increased reactive oxygen species (ROS) formation have been implicated in the aging process. ${ }^{11}$ Clearly, autophagic removal of defective mitochondria is of crucial importance for cell survival. Inhibition of autophagy by 3-MA in growth-arrested human fibroblasts, as a model of cell aging, results in the accumulation of lipofuscin-like material and of mitochondria, with a lowmembrane potential. ${ }^{62}$

As discussed above, we predict that autophagy is increased in type II diabetes. In most cases, this type of diabetes is caused by the inability of the body to buffer the free fatty acid 
concentration. This increases the redox pressure on the mitochondrial respiratory chain, increases ROS production, reduces mitochondrial function, and increases apoptosis, for example, of $\beta$-cells. ${ }^{63}$ Palmitate is the fatty acid that is most potent in causing insulin resistance. As palmitate is the precursor of ceramide and sphingolipid biosynthesis, the sphingolipid pathway has been implicated in the etiology of insulin resistance. ${ }^{64}$ Interestingly, ceramide has been shown to activate autophagy by upregulating beclin1 and inhibiting $\mathrm{PKB} ;{ }^{65}$ a decrease in intracellular amino-acid concentrations and a decrease in mTOR-dependent signaling may also have contributed to the activation of autophagy by ceramide, although the link with autophagy was not studied. ${ }^{66}$ If autophagy turns out to be elevated in type II diabetes, one can also speculate that the onset of insulin resistance in elderly people ${ }^{67}$ could in fact be an adaptive mechanism intended to increase autophagy, which helps to boost the ability to remove damaged mitochondria (and presumably other damaged cellular structures).

\section{Autophagy and Cell Death}

Autophagic cell death or type-Il programmed cell death is distinct from apoptosis, or type-I programmed cell death. ${ }^{16}$ However, the relationship between autophagy and apoptosis is actually probably more complex, because autophagy is not only able to collaborate with apoptosis to produce cell death (for reviews, see Debnath et al. ${ }^{14}$ and Baehrecke ${ }^{15}$ ) but, as we have seen in the preceding section, it can also act as a survival mechanism. From the recent literature, it has become clear that prolonged autophagy in the absence of the central core of the apoptotic machinery (bax/Bak-/- cells) is a cell survival mechanism that delays cell death in hematopoietic cells when growth factors and nutrients are in short supply. ${ }^{22}$ However, in the context of the same genetic background, embryonic fibroblasts utilize the autophagic machinery to die in response to apoptotic inducers. ${ }^{68}$

We will review our understanding of the control of autophagy during cell death, and will point out similarities to and differences from the regulation of autophagy observed during cell survival.

\section{Targeting the class-I PI3K and TOR pathways during programmed autophagy and autophagic cell death}

In Drosophila, a marked increase in autophagy is observed at the end of the larval stage. ${ }^{15}$ This developmental programmed autophagy is hormonally controlled by ecdysone, and is responsible for the elimination of organs, such as the fat body, during metamorphosis. The increase in autophagy of the fat body is caused by the inhibition of the class-I PI3K pathway by ecdysone. ${ }^{69}$ The inhibitory effect is observed when the ecdysone receptor is expressed. How ecdysone and its receptor inhibit the class-I PI3K pathway remains to be investigated. It is interesting to note that programmed autophagy does not depend on the feeding status, and inhibition of TOR signaling can further increase autophagy. This observation led the authors to suggest that dTOR may be only partially inhibited during programmed autophagy or possibly not involved at all. Overall, this study demonstrates the physiological significance of class-I PI3K during programmed autophagy. It also reveals the cross-talk between two signaling pathways involved in controlling autophagy, that is, the class-I PI3K and the ecdysone-receptor signaling.

The antiestrogen tamoxifen induces autophagy and cell death in MCF-7 cells. ${ }^{70}$ Tamoxifen stimulates autophagy by increasing the intracellular level of ceramide and abolishing the inhibitory effect of the class-I PI3K pathway on autophagy. ${ }^{65}$ Unexpectedly, however, in a rat cardiomyocytederived cell line, the activation of class-I PI3K during glucose deprivation induces the accumulation of autophagic vacuoles and causes cell death. ${ }^{52} \mathrm{~A}$ further degree of complexity is introduced by the observation that in glioma cells containing a Ras mutant (RasG12V) in which several signaling pathways, including the class-I PI3K pathway, were stimulated, autophagic cell death was induced. ${ }^{71}$ In contrast, the expression of a Ras mutant (RasG12V, Y40C) that specifically activates class-I PI3K inhibits starvation-induced autophagy in transformed fibroblasts, ${ }^{72}$ which fits in with the inhibitory role of class-I PI3K on autophagy in other models (see above).

\section{Other signaling pathways activated during autophagic cell death}

The death-associated protein kinase (DAPK) family DAPK and its related kinase death-associated related protein kinase-1 (DRP-1) are $\mathrm{Ca}^{2+} /$ calmodulin-regulated kinases, which act as positive effectors of cell death through caspasedependent apoptosis induced in response to various stimuli, such as interferon- $\gamma, \operatorname{TNF} \alpha$, and TGF $\beta$, and loss of interaction with the extracellular matrix. ${ }^{73}$ The phosphorylation of myosin-light chain mediates membrane blebbing during cell death. Expression of activated forms of DAP kinase and DRP1 trigger autophagy and cell death independently of caspase activity in carcinoma cells with nonfunctional p53. ${ }^{74}$ Moreover, the expression of a dominant-negative form of DRP-1 blocked the induction of autophagy and cell death in MCF-7 in response to tamoxifen treatment. It remains to be determined whether DRP-1 and ceramide act in the same signaling pathway, and contribute to tamoxifen-induced autophagy in MCF-7 cells. In this context, it is important to note that ceramide stimulated the expression of DAP kinase in neuronal cells. ${ }^{75}$ Interestingly, DRP-1 was found to be associated with the lumen of autophagosomes in HEK293 cells, suggesting that this kinase can phosphorylate some elements of the molecular machinery involved in the formation of autophagosomes. ${ }^{74}$ It is striking that DRP-1 also controls amino-acidsensitive autophagy in MCF-7 cells. This suggests that similar control mechanisms operate during starvation-induced autophagy and autophagic cell death. It remains an open question as to whether autophagic cell death and starvation-induced autophagy are controlled by different domains of DRP-1, and subsequently by different signaling pathways.

TNF-related apoptosis-inducing factor (TRAIL) and the TRAIL receptor

It has been shown recently that both apoptosis and autophagy contribute to the formation of hollow acini-like structures in an 
in-vitro model of mammary gland morphogenesis. ${ }^{76}$ TRAILdependent autophagy was blocked in cells expressing either truncated TRAIL receptors or a dominant-negative form of the Fas-associated death domain protein (FADD), which is required to recruit the apoptosis initiator procaspase-8. ${ }^{76,77}$ Interestingly, the death domain of FADD can activate a cell death pathway involving both apoptosis and autophagy that is selectively inactivated at the earliest stages of epithelial cancer development. ${ }^{77}$

$\mathrm{TNF} \alpha$, another member of the apoptosis-inducing family, stimulates autophagy and apoptosis in T-lymphoblastic leukemia cells. ${ }^{78}$ Inhibition of autophagy by 3-MA protects these cells against death. In addition, TNF $\alpha$ stimulates autophagic cell death independently of caspase activation. ${ }^{79}$ An analysis of gene expression in Drosophila suggests that the TNF $\alpha$-like pathway is involved during autophagic cell death in salivary glands. ${ }^{80}$ Recently, the interaction of Atg5 with the death domain of FADD has been shown to play a crucial role in interferon- $\gamma$-induced cell death independently of detectable activation of caspase-8..$^{81}$ Elucidation of signaling events downstream of FADD in Atg5-induced cell death would contribute to a better understanding of the control of autophagic cell death.

Jun N-terminal kinase (JNK)

Autophagic cell death has been observed in fibroblasts and monocytoid cells in response to the inhibition of caspase- $8{ }^{82}$ The accumulation of autophagic vacuoles is dependent on receptor-interacting protein (RIP), a protein associated with the cytoplasmic domain of the death receptor, and on the activation of JNK and its upstream kinase, MKK7. In addition, RIP is a substrate for caspase- 8 , which cleaves and inactivates it. This study suggests that caspase- 8 may also have a role in processes other than apoptosis. Another interesting observation is the implication of c-Jun, a target of JNK, in the control of autophagic cell death, which suggests that transcriptional activity is required to induce cell death, and also that de novo protein synthesis is required under these conditions. This is supported by the observation that cell death is prevented in the presence of cycloheximide. However, the nature of the genes induced remains to be determined. Genome-wide scale investigations have shown that, during autophagic cell death in Drosophila, the expression of a large array of genes, including several ATG genes (ATG2, 4-7, and 12), is upregulated. ${ }^{80,83}$ Accumulation of ATGs has also been observed in mammalian cells undergoing autophagic cell death, and is dependent on the expression of proteins of the $\mathrm{Bcl}-2$ family (Bcl-2 and $\mathrm{Bc} 1-\mathrm{xL}){ }^{68}$ An accumulation of Beclin 1 has been reported during tamoxifen-induced autophagy. ${ }^{65}$ The upregulation of ATG proteins is probably important in determining the amplitude of the autophagic response. The upregulation of Atg proteins is not a common trait during starvation-induced autophagy. ${ }^{84}$ The initiation of autophagy may not in fact depend on protein synthesis (for a discussion, see Abeliovich et al. ${ }^{85}$ ).

\section{Conclusion}

The dual nature of autophagy, which is a mechanism involved in both cell survival and cell death, has been known since this catabolic pathway was discovered in mammalian cells. ${ }^{23}$ It is clear that the detection of autophagosomes in dying cells is not sufficient to incriminate autophagy as a mechanism of cell death, because they may accumulate not due to activation of the flux through the autophagic pathway, but rather as a result of inhibited processing of autophagosomes. (Likewise, the accumulation of autophagosomes in healthy cells may not always match an increase in autophagic flux.) The demonstration that activation of the autophagic machinery, accompanied by increased flux, can result in the death of cells that are not bioenergetically compromised would be a strong indication that autophagy is indeed a cell death mechanism in its own right, but this has yet to be demonstrated. ${ }^{53}$ However, the fact that the molecular machinery of autophagy is required during cell death has been recently demonstrated by genetic methods. ${ }^{68,82}$ Whereas starvation-induced autophagy is a mechanism tightly controlled by amino acids and hormones, it appears that greater complexity of the signaling pathways, which overlap with those of apoptotic signaling, is observed during cell death. Care must be exercised in attempting to interpret the effects of 3-MA during cell death, because this compound, in addition to its ability to inhibit class-III PI3K, which is involved in the formation of autophagosomes, ${ }^{27}$ can also affect other signaling components, such as class-I $\mathrm{PI}^{26,27}$ and MAP kinases (JNK, p38). ${ }^{86}$ The accumulation of autophagy gene products during cell death, and their relationship with members of the Bcl-2 family, is a promising track to explore in order to obtain a better understanding of how autophagy is controlled during cell death. ${ }^{68,87}$ The interaction between Beclin 1 and $\mathrm{Bcl}-2 / \mathrm{Bcl}-\mathrm{xL}^{88}$ is probably a key event in turning the accumulation of autophagosomes on or off. 68

Mitochondria play a central role in the execution of apoptosis by releasing apoptotic factors. ${ }^{89}$ This organelle is also a target for the autophagic pathway. ${ }^{17}$ The autophagic elimination of apoptotic-competent mitochondria is clearly a cell defense mechanism. Starvation-induced autophagy can proceed in the absence of the selective elimination of mitochondria. ${ }^{90}$ Unraveling the molecular basis of mitochondrion-specific autophagy would be a crucial step in our understanding of the role of autophagy in controlling cellular homeostasis. It could also be expected to yield more insight into complicated processes such as aging, type II diabetes, and various other disorders.

\section{Acknowledgements}

We would like to apologize to any authors whose work could not be cited here because of space limitation. Work in P Codogno's laboratory is supported by institutional funding from The Institut National de la Santé et de la Recherche Médicale (INSERM) and grants from the Association pour la Recherche sur le Cancer (ARC 3503).

\section{References}

1. Dunn Jr WA (1994) Autophagy and related mechanisms of lysosome-mediated protein degradation. Trends Cell Biol. 4: 139-143

2. Schwartz AL and Ciechanover A (1999) The ubiquitin-proteasome pathway and pathogenesis of human diseases. Annu. Rev. Med. 50: 57-74 
3. Majeski AE and Dice JF (2004) Mechanisms of chaperone-mediated autophagy. Int. J. Biochem. Cell Biol. 36: 2435-2444

4. Cuervo AM (2004) Autophagy: in sickness and in health. Trends Cell Biol. 14 70-77

5. Eskelinen EL (2005) Maturation of autophagic vacuoles in mammalian cells Autophagy 1: 1-10

6. Klionsky DJ, Cregg JM, Dunn Jr WA, Emr SD, Sakai Y, Sandoval IV, Sibirny A, Subramani S, Thumm M, Veenhuis M and Ohsumi Y (2003) A unified nomenclature for yeast autophagy-related genes. Dev. Cell 5: 539-545

7. Mizushima N, Yoshimori T and Ohsumi Y (2003) Role of the Apg12 conjugation system in mammalian autophagy. Int. J. Biochem. Cell Biol. 35: 553-561

8. Meijer AJ and Codogno P (2004) Regulation and role of autophagy in mammalian cells. Int. J. Biochem. Cell Biol. 36: 2445-2462

9. Levine B and Klionsky DJ (2004) Development by self-digestion: molecular mechanisms and biological functions of autophagy. Dev. Cell 6: 463-477

10. Blommaart EF, Luiken JJ, Blommaart PJ, van Woerkom GM and Meijer AJ (1995) Phosphorylation of ribosomal protein S6 is inhibitory for autophagy in isolated rat hepatocytes. J. Biol. Chem. 270: 2320-2326

11. Bergamini E, Cavallini G, Donati A and Gori Z (2004) The role of macroautophagy in the ageing process, anti-ageing intervention and ageassociated diseases. Int. J. Biochem. Cell Biol. 36: 2392-2404

12. Lum JJ, DeBernardinis RJ and Thompson CB (2005) Autophagy in metazoans: cell survival in the land of plenty. Nat. Rev. Mol. Cell Biol. 6: 439-448

13. Shintani T and Klionsky DJ (2004) Autophagy in health and disease: a doubleedged sword. Science 306: 990-995

14. Debnath J, Baehrecke EH and Kroemer G (2005) Does autophagy contribute to cell death? Autophagy 1: 10-18

15. Baehrecke EH (2005) Autophagy: dual roles in life and death? Nat. Rev. Mol. Cell Biol. 6: 505-510

16. Clarke PG (1990) Developmental cell death: morphological diversity and multiple mechanisms. Anat. Embryol. 181: 195-213

17. Rodriguez-Enriquez S, He L and Lemasters JJ (2004) Role of mitochondrial permeability transition pores in mitochondrial autophagy. Int. J. Biochem. Cell Biol. 36: 2463-2472

18. Kuma A, Hatano M, Matsui M, Yamamoto A, Nakaya H, Yoshimori T, Ohsum $\mathrm{Y}$, Tokuhisa T and Mizushima N (2004) The role of autophagy during the early neonatal starvation period. Nature 432: 1032-1036

19. Mizushima N, Yamamoto A, Matsui M, Yoshimori T and Ohsumi Y (2004) In vivo analysis of autophagy in response to nutrient starvation using transgenic mice expressing a fluorescent autophagosome marker. Mol. Biol. Cell 15: $1101-1111$

20. Ogier-Denis E and Codogno P (2003) Autophagy: a barrier or an adaptive response to cancer? Biochim. Biophys. Acta 1603: 113-128

21. Boya P, Gonzalez-Polo RA, Casares N, Perfettini JL, Dessen P, Larochette N, Metivier D, Meley D, Souquere S, Yoshimori T, Pierron G, Codogno P and Kroemer G (2005) Inhibition of macroautophagy triggers apoptosis. Mol. Cell. Biol. 25: 1025-1040

22. Lum JJ, Bauer DE, Kong M, Harris MH, Li C, Lindsten T and Thompson CB (2005) Growth factor regulation of autophagy and cell survival in the absence of apoptosis. Cell 120: 237-248

23. de Duve C and Wattiaux R (1966) Functions of lysosomes. Annu. Rev. Physiol. 28: 435-492

24. Mortimore GE, Miotto G, Venerando R and Kadowaki M (1996) Autophagy. In Biology of the Lysosome Lloyd JB and Mason RW (eds) (New York: Plenum Press) pp. 93-135

25. Holen I, Gordon PB and Seglen PO (1993) Inhibition of hepatocytic autophagy by okadaic acid and other protein phosphatase inhibitors. Eur. J. Biochem. 215: 113-122

26. Blommaart EF, Krause U, Schellens JP, Vreeling-Sindelarova $\mathrm{H}$ and Meijer AJ (1997) The phosphatidylinositol 3-kinase inhibitors wortmannin and LY294002 inhibit autophagy in isolated rat hepatocytes. Eur. J. Biochem. 243: 240-246

27. Petiot A, Ogier-Denis E, Blommaart EF, Meijer AJ and Codogno P (2000) Distinct classes of phosphatidylinositol $3^{\prime}$-kinases are involved in signaling pathways that control macroautophagy in HT-29 cells. J. Biol. Chem. 275 992-998

28. Arico S, Petiot A, Bauvy C, Dubbelhuis PF, Meijer AJ, Codogno P and OgierDenis E (2001) The tumor suppressor PTEN positively regulates macroautophagy by inhibiting the phosphatidylinositol 3-kinase/protein kinase B pathway. J. Biol. Chem. 276: 35243-35246
29. Kihara A, Noda T, Ishihara N and Ohsumi $Y$ (2001) Two distinct Vps34 phosphatidylinositol 3-kinase complexes function in autophagy and carboxypeptidase $Y$ sorting in Saccharomyces cerevisiae. J. Cell Biol. 152: $519-530$

30. Suzuki K, Kirisako T, Kamada Y, Mizushima N, Noda T and Ohsumi Y (2001) The pre-autophagosomal structure organized by concerted functions of APG genes is essential for autophagosome formation. EMBO J. 20: 5971-5981

31. Liang XH, Jackson S, Seaman M, Brown K, Kempkes B, Hibshoosh H and Levine B (1999) Induction of autophagy and inhibition of tumorigenesis by beclin 1. Nature 402: $672-676$

32. Kihara A, Kabeya $Y$, Ohsumi $Y$ and Yoshimori $T$ (2001) Beclinphosphatidylinositol 3-kinase complex functions at the trans-Golgi network. EMBO Rep. 2: 330-335

33. van Sluijters DA, Dubbelhuis PF, Blommaart EFC and Meijer AJ (2000) Aminoacid-dependent signal transduction. Biochem. J. 351: 545-550

34. Cheng SW, Fryer LG, Carling D and Shepherd PR (2004) Thr2446 is a novel mammalian target of rapamycin (mTOR) phosphorylation site regulated by nutrient status. J. Biol. Chem. 279: 15719-15722

35. Inoki K, Corradetti MN and Guan KL (2005) Dysregulation of the TSC-mTOR pathway in human disease. Nat. Genet. 37: 19-24

36. Smith EM, Finn SG, Tee AR, Browne GJ and Proud CG (2005) The tuberous sclerosis protein TSC2 is not required for the regulation of the mammalian target of rapamycin by amino acids and certain cellular stresses. J. Biol. Chem. 280: $18717-18727$

37. Talloczy Z, Jiang W, Virgin HWt, Leib DA, Scheuner D, Kaufman RJ, Eskelinen EL and Levine $B$ (2002) Regulation of starvation- and virus-induced autophagy by the elF2alpha kinase signaling pathway. Proc. Natl. Acad. Sci. USA 99: 190-195

38. Jacinto $E$ and Hall MN (2003) Tor signalling in bugs, brain and brawn. Nat. Rev. Mol. Cell Biol. 4: 117-126

39. Meijer AJ and Dubbelhuis PF (2004) Amino acid signalling and the integration of metabolism. Biochem. Biophys. Res. Commun. 313: 397-403

40. Kanazawa T, Taneike I, Akaishi R, Yoshizawa F, Furuya N, Fujimura S and Kadowaki M (2004) Amino acids and insulin control autophagic proteolysis through different signaling pathways in relation to $\mathrm{mTOR}$ in isolated rat hepatocytes. J. Biol. Chem. 279: 8452-8459

41. Beugnet A, Tee AR, Taylor PM and Proud CG (2003) Regulation of targets of mTOR (mammalian target of rapamycin) signalling by intracellular amino acid availability. Biochem. J. 372: 555-566

42. Mordier S, Deval C, Bechet D, Tassa A and Ferrara M (2000) Leucine limitation induces autophagy and activation of lysosome-dependent proteolysis in $\mathrm{C} 2 \mathrm{C} 12$ myotubes through a mammalian target of rapamycin-independent signaling pathway. J. Biol. Chem. 275: 29900-29906

43. Tassa A, Roux MP, Attaix D and Bechet DM (2003) Class III phosphoinositide 3-kinase-Beclin1 complex mediates the amino acid-dependent regulation of autophagy in $\mathrm{C} 2 \mathrm{C} 12$ myotubes. Biochem. J. 376: 577-586

44. Sarbassov DD, Guertin DA, Ali SM and Sabatini DM (2005) Phosphorylation and regulation of Akt/PKB by the rictor-mTOR complex. Science 307: 1098-1101

45. Pattingre $S$, Bauvy $C$ and Codogno $P$ (2003) Amino acids interfere with the ERK $1 / 2$-dependent control of macroautophagy by controlling the activation of Raf-1 in human colon cancer HT-29 cells. J. Biol. Chem. 278: 16667-16674

46. vom Dahl S, Schliess F, Reissmann R, Gorg B, Weiergraber O, Kocalkova M, Dombrowski $F$ and Haussinger D (2003) Involvement of integrins in osmosensing and signaling toward autophagic proteolysis in rat liver. J. Biol. Chem. 278: 27088-27095

47. Dennis PB, Jaeschke A, Saitoh M, Fowler B, Kozma SC and Thomas G (2001) Mammalian TOR: a homeostatic ATP sensor. Science 294: 1102-1105

48. Hardie DG (2003) The AMP-activated protein kinase cascade: the key sensor of cellular energy status. Endocrinology 144: 5179-5183

49. Wang Z, Wilson WA, Fujino MA and Roach PJ (2001) Antagonistic controls of autophagy and glycogen accumulation by Snf1p, the yeast homolog of AMP-activated protein kinase, and the cyclin-dependent kinase Pho85p. Mol. Cell. Biol. 21: 5742-5752

50. Samari HR and Seglen PO (1998) Inhibition of hepatocytic autophagy by adenosine, aminoimidazole-4-carboxamide riboside, and N6-mercaptopurine riboside. Evidence for involvement of amp-activated protein kinase. J. Biol. Chem. 273: 23758-23763 
51. Moller MT, Samari HR and Seglen PO (2004) Toxin-induced tail phosphorylation of hepatocellular S6 kinase: evidence for a dual involvement of the AMP-activated protein kinase in S6 kinase regulation. Toxicol. Sci. 82: 628-637

52. Aki T, Yamaguchi K, Fujimiya T and Mizukami Y (2003) Phosphoinositide 3-kinase accelerates autophagic cell death during glucose deprivation in the rat cardiomyocyte-derived cell line H9c2. Oncogene 22: 8529-8535

53. Edinger AL and Thompson CB (2004) Death by design: apoptosis, necrosis and autophagy. Curr. Opin. Cell Biol. 16: 663-669

54. Apfeld J, O'Connor G, McDonagh T, DiStefano PS and Curtis R (2004) The AMP-activated protein kinase AAK-2 links energy levels and insulin-like signals to lifespan in C. elegans. Genes Dev. 18: 3004-3009

55. Arad M, Benson DW, Perez-Atayde AR, McKenna WJ, Sparks EA, Kanter RJ, McGarry K, Seidman JG and Seidman CE (2002) Constitutively active AMP kinase mutations cause glycogen storage disease mimicking hypertrophic cardiomyopathy. J. Clin. Invest. 109: 357-362

56. Um SH, Frigerio F, Watanabe M, Picard F, Joaquin M, Sticker M, Fumagalli S, Allegrini PR, Kozma SC, Auwerx J and Thomas G (2004) Absence of S6K1 protects against age- and diet-induced obesity while enhancing insulin sensitivity. Nature 431: 200-205

57. Khamzina L, Veilleux A, Bergeron S and Marette A (2005) Increased activation of the mammalian target of rapamycin pathway in liver and skeletal muscle of obese rats: possible involvement in obesity-linked insulin resistance. Endocrinology 146: 1473-1481

58. Klionsky DJ, Meijer AJ, Codogno P, Neufeld TP and Scott RC (2005) Autophagy and p70S6 kinase. Autophagy 1: 59-61

59. Scott RC, Schuldiner $O$ and Neufeld TP (2004) Role and regulation of starvation-induced autophagy in the Drosophila fat body. Dev. Cell 7: 167-178

60. Katic M and Kahn CR (2005) The role of insulin and IGF-1 signaling in longevity. Cell Mol. Life Sci. 62: 320-343

61. Melendez A, Talloczy Z, Seaman M, Eskelinen EL, Hall DH and Levine B (2003) Autophagy genes are essential for dauer development and life-span extension in C. elegans. Science 301: 1387-1391

62. Stroikin Y, Dalen H, Loof S and Terman A (2004) Inhibition of autophagy with 3-methyladenine results in impaired turnover of lysosomes and accumulation of lipofuscin-like material. Eur. J. Cell Biol. 83: 583-590

63. Evans JL, Goldfine ID, Maddux BA and Grodsky GM (2002) Oxidative stress and stress-activated signaling pathways: a unifying hypothesis of type 2 diabetes. Endocr. Rev. 23: 599-622

64. Tagami S, Inokuchi Ji J, Kabayama K, Yoshimura H, Kitamura F, Uemura S, Ogawa C, Ishii A, Saito M, Ohtsuka Y, Sakaue S and Igarashi Y (2002) Ganglioside GM3 participates in the pathological conditions of insulin resistance. J. Biol. Chem. 277: 3085-3092

65. Scarlatti F, Bauvy C, Ventruti A, Sala G, Cluzeaud F, Vandewalle A, Ghidoni R and Codogno $P$ (2004) Ceramide-mediated macroautophagy involves inhibition of protein kinase B and up-regulation of beclin 1. J. Biol. Chem. 279: 18384-18391

66. Hyde R, Hajduch E, Powell DJ, Taylor PM and Hundal HS (2005) Ceramide down-regulates System A amino acid transport and protein synthesis in rat skeletal muscle cells. FASEB J. 19: 461-463

67. Petersen KF, Befroy D, Dufour S, Dziura J, Ariyan C, Rothman DL, DiPietro L, Cline GW and Shulman GI (2003) Mitochondrial dysfunction in the elderly: possible role in insulin resistance. Science 300: 1140-1142

68. Shimizu S, Kanaseki T, Mizushima N, Mizuta T, Arakawa-Kobayashi S, Thompson CB and Tsujimoto Y (2004) Role of Bcl-2 family proteins in a nonapoptotic programmed cell death dependent on autophagy genes. Nat. Cell Biol. 6: 1221-1228

69. Rusten TE, Lindmo K, Juhasz G, Sass M, Seglen PO, Brech A and Stenmark H (2004) Programmed autophagy in the Drosophila fat body is induced by ecdysone through regulation of the PI3K pathway. Dev. Cell 7: 179-192

70. Bursch W, Ellinger A, Kienzl H, Torok L, Pandey S, Sikorska M, Walker R and Hermann RS (1996) Active cell death induced by the anti-estrogens tamoxifen and ICI 164384 in human mammary carcinoma cells (MCF-7) in culture: the role of autophagy. Carcinogenesis 17: 1595-1607

71. Chi S, Kitanaka C, Noguchi K, Mochizuki T, Nagashima Y, Shirouzu M, Fujita H, Yoshida M, Chen W, Asai A, Himeno M, Yokoyama S and Kuchino Y (1999)
Oncogenic Ras triggers cell suicide through the activation of a caspaseindependent cell death program in human cancer cells. Oncogene 18: 2281-2290

72. Furuta S, Hidaka E, Ogata A, Yokota S and Kamata T (2004) Ras is involved in the negative control of autophagy through the class I PI3-kinase. Oncogene 23: 3898-3904

73. Shohat G, Spivak-Kroizman T, Eisenstein M and Kimchi A (2002) The regulation of death-associated protein (DAP) kinase in apoptosis. Eur. Cytokine Netw. 13: 398-400

74. Inbal B, Bialik S, Sabanay I, Shani G and Kimchi A (2002) DAP kinase and DRP-1 mediate membrane blebbing and the formation of autophagic vesicles during programmed cell death. J. Cell Biol. 157: 455-468

75. Pelled D, Raveh T, Riebeling C, Fridkin M, Berissi H, Futerman AH and Kimchi A (2002) Death-associated protein (DAP) kinase plays a central role in ceramide-induced apoptosis in cultured hippocampal neurons. J. Biol. Chem. 277: 1957-1961

76. Mills KR, Reginato M, Debnath J, Queenan B and Brugge JS (2004) Tumor necrosis factor-related apoptosis-inducing ligand (TRAIL) is required for induction of autophagy during lumen formation in vitro. Proc. Natl. Acad. Sci. USA 101: 3438-3443

77. Thorburn J, Moore F, Rao A, Barclay WW, Thomas LR, Grant KW, Cramer SD and Thorburn A (2005) Selective inactivation of a Fas-associated death domain protein (FADD)-dependent apoptosis and autophagy pathway in immortal epithelial cells. Mol. Biol. Cell. 16: 1189-1199

78. Jia L, Dourmashkin RR, Allen PD, Gray AB, Newland AC and Kelsey SM (1997) Inhibition of autophagy abrogates tumour necrosis factor alpha induced apoptosis in human T-lymphoblastic leukaemic cells. Br. J. Haematol. 98: 673-685

79. Yanagisawa H, Miyashita T, Nakano $Y$ and Yamamoto D (2003) HSpin1, a transmembrane protein interacting with $\mathrm{Bcl}-2 / \mathrm{Bcl}-\mathrm{xL}$, induces a caspaseindependent autophagic cell death. Cell Death Differ. 10: 798-807

80. Gorski SM, Chittaranjan S, Pleasance ED, Freeman JD, Anderson CL, Varhol RJ, Coughlin SM, Zuyderduyn SD, Jones SJ and Marra MA (2003) A SAGE approach to discovery of genes involved in autophagic cell death. Curr. Biol. 13: 358-363

81. Pyo JO, Jang MH, Kwon YK, Lee HJ, Jun Jl, Woo HN, Cho DH, Choi B, Lee H, Kim JH, Mizushima N, Oshumi Y and Jung YK (2005) Essential roles of Atg5 and FADD in autophagic cell death: dissection of autophagic cell death into vacuole formation and cell death. J. Biol. Chem. 280: 20722-20729

82. Yu L, Alva A, Su H, Dutt P, Freundt E, Welsh S, Baehrecke EH and Lenardo MJ (2004) Regulation of an ATG7-beclin 1 program of autophagic cell death by caspase-8. Science 304: 1500-1502

83. Lee CY, Clough EA, Yellon P, Teslovich TM, Stephan DA and Baehrecke EH (2003) Genome-wide analyses of steroid- and radiation-triggered programmed cell death in Drosophila. Curr. Biol. 13: 350-357

84. Peng T, Golub TR and Sabatini DM (2002) The immunosuppressant rapamycin mimics a starvation-like signal distinct from amino acid and glucose deprivation. Mol. Cell. Biol. 22: 5575-5584

85. Abeliovich H, Dunn Jr WA, Kim J and Klionsky DJ (2000) Dissection of autophagosome biogenesis into distinct nucleation and expansion steps. J. Cell Biol. 151: 1025-1034

86. Xue L, Fletcher GC and Tolkovsky AM (1999) Autophagy is activated by apoptotic signalling in sympathetic neurons: an alternative mechanism of death execution. Mol. Cell Neurosci. 14: 180-198

87. Saeki K, Yuo A, Okuma E, Yazaki Y, Susin SA, Kroemer G and Takaku F (2000) Bcl-2 down-regulation causes autophagy in a caspase-independent manner in human leukemic HL60 cells. Cell Death Differ. 7: 1263-1269

88. Liang XH, Kleeman LK, Jiang HH, Gordon G, Goldman JE, Berry G, Herman B and Levine B (1998) Protection against fatal Sindbis virus encephalitis by beclin, a novel Bcl-2-interacting protein. J. Virol. 72: 8586-8596

89. Brenner $C$ and Kroemer $G$ (2000) Apoptosis. Mitochondria - the death signal integrators. Science 289: 1150-1151

90. Kissova I, Deffieu M, Manon S and Camougrand N (2004) Uth1p is involved in the autophagic degradation of mitochondria. J. Biol. Chem. 279 . 39068-39074 